\title{
Preoperative habituation and effects of prefrontal lesions on an autonomic response of the rat
}

\author{
R. C. WILCOTT \\ Case Western Reserve University, Cleveland, Ohio
}

\begin{abstract}
Skin potential responses to an auditory stimulus were increased in amplitude following bilateral prefrontal lesions in the rat. If skin potential responses were habituated across days before surgery, however, the effect of prefrontal lesions was considerably reduced. Results of two control experiments indicated that this finding was probably not due to extraneous factors. These results agree with earlier observations on heart rate by Glaser and Griffin (1962). It is concluded that although the prefrontal cortex normally maintains inhibitory control over autonomic responses, during habituation of an autonomic response this inhibitory control tends to shift from the prefrontal cortex to other parts of the brain. It was further found that after the amplitudes of SP responses are increased by prefrontal lesions, they will habituate. This suggests that the prefrontal cortex is not essential for habituation.
\end{abstract}

The activity of autonomic variables such as heart rate and skin potential is usually increased following bilateral one-stage lesions in the frontal cortex, including the prefrontal region, in various animals that have been tested (Brutkowski, 1965; Glaser \& Griffin, 1962; Murray \& Wang, 1967; Wilcott, 1967). These results seem to indicate that the prefrontal and adjacent parts of the cortex normally maintain an inhibitory influence on the autonomic nervous system. This conclusion is further supported by the observation that electrical stimulation in or near the prefrontal region will suppress heart rate (Glaser \& Griffin, 1962) and skin potential responses (Wang \& Brown, 1956; Wilcott, 1974).

Effects of frontal lesions on autonomic activity, however, can apparently be modified by the animal's experience before surgery. Glaser and Griffin (1962) have found that following medial frontal lesions in the rat, the heart rate response did not habituate to a noxious stimulus (tail immersed into cold water). But if, before surgery, this noxious stimulus was presented daily over 10 days until the heart rate response was largely habituated, the habituation was maintained with 6 of 8 rats tested 7 days after frontal lesions.

This problem may be important in our understanding of the inhibitory mechanisms in the frontal cortex. It was decided, therefore, to further investigate

This research was supported by U.S. Public Health Service Grant MH-31916 from the National Institute of Mental Health. Thanks are extended to Janet Nelson, Beth Stein, Betty Wentworth, and George Franklyn for assistance. Request for reprints should be sent to R. C. Wilcott, Department of Psychology, Case Western Reserve University, Cleveland, Ohio 44106. the influence of preoperative habituation of an autonomic response on effects produced by frontal lesions in the rat. The skin potential (SP) was used as the measure of autonomic activity.

Several methods of restraining rats during SP recording were tested, including strapping them onto a shaped Plexiglas platform (Roberts \& Young, 1971). It was found, however, that these methods usually produced a relatively high frequency of nonspecific SP responses and little habituation of the SP response to the auditory stimulus used. The method adopted was to have the experimenter or another person hold the rat against his body with both hands while sitting on a high stool. For reasons given below, this procedure was employed in two different ways and two separate experiments are reported. Data from a third experiment appear to show that the reduction in SP response amplitude across days in Experiments 1 and 2 was due to habituation and not to some extraneous factor.

\section{EXPERIMENT 1}

In Experiment 1, the experimenter held all rats both before and after surgery.

\section{Method}

Subjects. The subjects were 35 Holtzman male rats, who ranged in weight from 350 to $385 \mathrm{~g}$ at the time of surgery.

Surgery. Following the procedure in the Glaser and Griffin (1962) study ${ }_{n}$ the lesions were predominantly in the medial frontal cortex. Measurements were made from the bregma, and the locations for bone removal were marked on both sides of the skull in an attempt to make lesions the same size across rats. Cortical tissue was removed by aspiration, using a fine pipette. Marks were made on the side of the pipette to serve as guides in determining the 
depth of lesions. Lesions appeared to include most of the prefrontal cortex as defined by projections from the mediodorsal nucleus of the thalamus (Krettek \& Price, 1977).

Sham-operated controls were tested in the same way, except that the dura was not removed.

All rats were given tetracycline in their drinking water for 5 days after surgery.

Histology. The rat was perfused through the heart, and the brain was removed. Lesions, as viewed from the dorsal surface, were marked on a standard chart (Figure 1). Frozen sections ( $40 \mu$ ) were then prepared, and the three sections closest to those shown in Figure 1 were photographed. Lesions from these cross sections were then marked on standard charts. The size of cortical lesions was expressed as a percentage value following procedures described in the Figure 1 caption.

SP Recording. SP was recorded from the plantar surface of the right hindfoot with the reference electrode on the tail. $\mathrm{AgAgCl}$ disk electrodes were mounted on grommets. The center opening of the grommet, $6 \mathrm{~mm}$ in diameter, was filled with a saline-bentonite paste. The polarization of electrodes was routinely tested by holding them together before being positioned on the rat. Polarization was always less than $2 \mathrm{mV}$. Electrodes were held in place on the rat's foot and tail by surgical tape. A small piece of masking tape was placed on the back of the rat's foot to aid removal of the surgical tape from this area. The tubercles at the plantar surface assisted the placement of electrodes at a relatively constant location across recording sessions. Recording was done with a Beckman Type $R$ Dynograph using a back EMF circuit to balance the SP level to the null point (Wilcott, 1958). SP responses were always uniphasic negative waves.

When holding a rat, the experimenter was in the same room with the recording equipment and about $6 \mathrm{f}$ from the equipment. The room was quiet, except for the constant hum of the chart drive and the room ventilation system, and was darkened except for a small lamp over the recording chart. An assistant operated the equipment.

An auditory stimulus was used to elicit SP responses. It consisted of a $2000-\mathrm{Hz}$ tone produced by a loudspeaker approximately 24 in. from the rat's head. Intensity was $56 \mathrm{~dB}\left(.002 \mathrm{dyne} / \mathrm{cm}^{2}\right)$. The stimulus was $1 \mathrm{sec}$ in duration and was controlled by a Hunter timer.

Procedure. The rats were handled daily by the experimenter for 14 days before SP recording. They were then adapted to the SP recording procedure during three daily 15 -min recording sessions. During these sessions, only SP nonspecific responses were recorded and the auditory stimulus was not presented.

Before the first experimental recording session, which was the next day after adaptation, the rats were divided into two groups, a preoperative habituation group and a preoperative nonhabituation group. These two groups were matched as far as possible on the basis of the amplitude of nonspecific responses during the last adaptation session. Preliminary observations on other rats had shown that the amplitudes of SP nonspecific responses were generally close to the amplitudes of the SP responses that would later be elicited by the auditory stimulus.

Each daily experimental session was about $15 \mathrm{~min}$ in duration, and the auditory stimulus was presented four times. Interstimulus interval was about 2 min. The stimulus was presented only when the rat was quiet and was not displaying a nonspecific SP response. Stimulus presentation was controlled by the assistant, and the experimenter did not know when the stimulus was to be presented. The amplitude of each of the four SP responses was measured and the mean taken. Occasionally, an SP response to the stimulus was obscured by movement artifact and it was necessary to repeat that stimulus trial. This occurred before and after surgery about equally often in both groups. A comparison of the mean SP response amplitudes on test days with and without this movement artifact indicated that repetition of a stimulus trial was not an important factor affecting differences between groups.
Preliminary observations on other rats had also shown that with some rats SP responses to the auditory stimulus could not be observed clearly, either because of a high rate of nonspecific responses or because responses were too low in amplitude. During the first experimental recording session, the rats had to meet two criteria. First, they had to display a low rate of nonspecific responses so that responses to the auditory stimulus could be observed clearly. Second, all SP responses to the auditory stimulus had to be at least $2 \mathrm{mV}$ in amplitude. Of a total of 48 rats used in the first experimental session, 35 met these criteria and were continued in the experiment. The remaining 13 rats were dropped from the study. At this time, the original matching of the preoperative habituation and nonhabituation groups on the basis of the amplitude of nonspecific responses was reviewed, and rats were shifted if necessary to maintain the matching as close as possible.

The preoperative nonhabituation group was then given one more experimental session, for a total of two daily experimental sessions, to provide a base level. They were operated on within 2 days. The preoperative habituation group was given 9 more experimental sessions for a total of 10 daily sessions. They were also operated on within 2 days after completion of testing. Before surgery, each group was divided into two subgroups, experimental and control The control subgroup received sham surgery.

Rats used in this experiment were not all tested at the same time, but were tested at four different times over approximately a 9 month period. Preoperative habituation and nonhabituation rats were included at each time of testing, and any unintended variation in procedures should have affected both groups to the same extent.

During preliminary testing with other rats, SP responses were recorded from one of the experimenter's fingers. It was found that he quickly habituated to the auditory stimulus during the first few sessions and thereafter displayed few SP nonspecific responses. This served to indicate that any SP responses of the experimenter would be unrelated to SP responses of the rat during experimental testing.

\section{Results}

Lesions. The lesions of the rat shown in Figure 1 were typical of all rats. The mean size of the lesions for the habituation experimental subgroup was $32.3 \%$. For the nonhabituation experimental subgroup, the mean was $31.8 \%$. Beginning 4 days after surgery and through to the end of the experiment, all rats were handled daily. They never resisted being picked up and displayed no signs of increased irritability.

SP responses. An example of an SP response to the auditory stimulus is given in Figure 2. The mean amplitudes of SP responses recorded before and after surgery for all subgroups are plotted in Figure 3.

As expected, prefrontal lesions increased the amplitude of the SP response of the nonhabituation experimental subgroup. Across Days 2 and 1 before surgery, the differences in SP response amplitudes of the nonhabituation experimental and control subgroups were not significant $[F(1,16)=1.21, p>.05]$. Across Days 7-13 after surgery, however, SP responses of the nonhabituation experimental subgroup were significantly larger than those of the control subgroup $[F(1,16)=8.75, p<.01]$.

After surgery, the nonhabituation experimental 


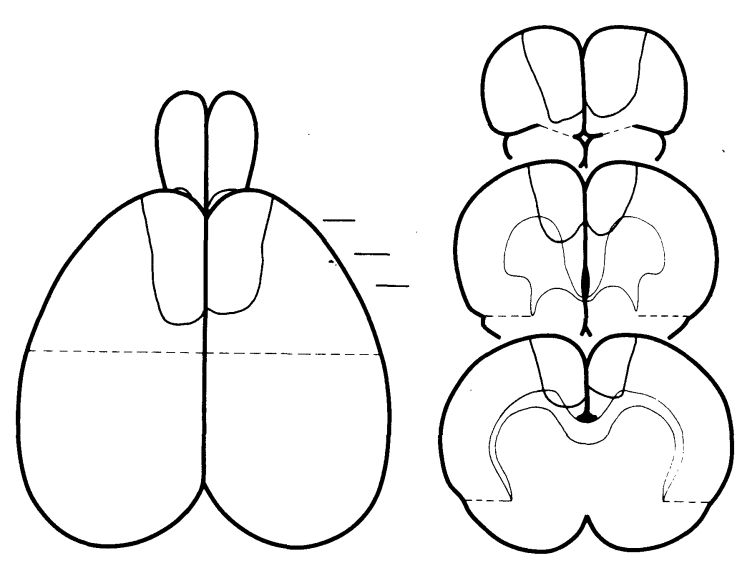

Figure 1. The extent of lesions in the cortex of one rat is marked on a standard dorsal chart and on standard cross-section charts. The locations of the cross sections are indicated at the dorsal chart. The area of lesions marked on the dorsal chart and the area of the total dorsal cortex anterior to the bregma (dotted line) were measured by a planimeter. The size of the lesions was then expressed as a percentage of the cortex anterior to the bregma. The area of lesions at each cross-section chart, excluding the part that extended into the corpus callosum, was measured in the same way, as was the area of the total cortex above the rhinal sulcus (dotted lines). The size of lesions at each of these cross sections was then expressed as a percentage of the total cortex in that cross section. A single percentage value was then taken as the mean of the dorsal and these three cross-section percentages. For this rat, it was $30.5 \%$. The extent of damage to the olfactory bulbs and corpus callosum was disregarded, but these structures recelved some damage in all rats and there appeared to be little difference in the extent of this damage between subgroups.

subgroups displayed significant habituation of SP responses across Days 7-13 $[\mathrm{F}(6,48)=2.93, \mathrm{p}<.05]$. Postoperative habituation of the nonhabituation control subgroup was also significant $[F(6,48)=4.59$, $\mathrm{p}<.01]$.

Differences in SP response amplitudes between the preoperative-habituation experimental and control subgroups across Days 10-1 before surgery were not significant $[F(1,15)=2.07, p>.05$, unweighted means procedure for unequal subgroup size]. The habituation experimental subgroup displayed significant habituation across Days 10-1 before surgery $[F(9,63)=4.48, p<.01]$. Habituation was also significant for the control subgroup $[F(9,64)=4.69$, $\mathrm{p}<.01]$.

Following surgery, differences between the habituation experimental and control subgroups across Days $7-13$ were not significant $[F(1,15)=2.59, p>$ $.05]$, although, as is apparent from Figure 3, during the early test days after surgery the amplitudes of the SP responses of the experimental subgroup were increased more than those of the control subgroup. Differences between these two subgroups across Days 7 and 8 were significant $[F(1,15)=5.73$, p $<$ $.05]$. These results demonstrate that prefrontal lesions disrupted the habituation of the experimental subgroup but that they rehabituated.

Although prefrontal lesions disrupted the habituation of the preoperative habituation experimental subgroup, Figure 3 shows that preoperative habituation did substantially reduce effects of prefrontal lesions on SP response amplitudes of this subgroup as compared with effects of prefrontal lesions on the nonhabituation experimental subgroup. This is documented by the following analyses. The differences between the amplitudes of SP responses of the nonhabituation experimental subgroup across Days 2 and 1 before surgery and those of the habituation experimental subgroup during the first 2 days of testing (10 and 9 ) before surgery did not reach significance $[F(1,15)=3.17, p>.05$, unweighted means procedure]. But differences in the amplitude of SP responses between these two subgroups across Days 713 after surgery were highly significant $[F(1,15)=$ 9.75, $\mathrm{p}<.01$ ]. Prefrontal lesions clearly had a greater effect on the nonhabituation experimental subgroup.

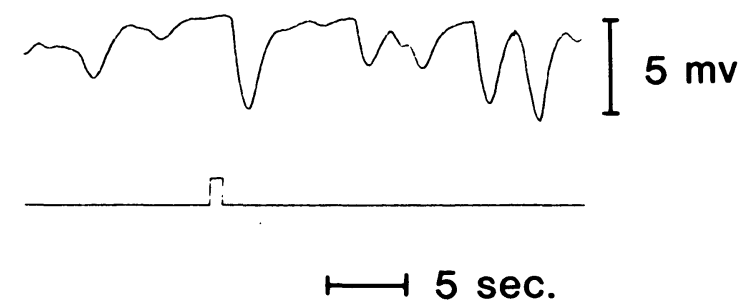

Figure 2. Typical SP response to the auditory stimulus and SP nonspecific responses.

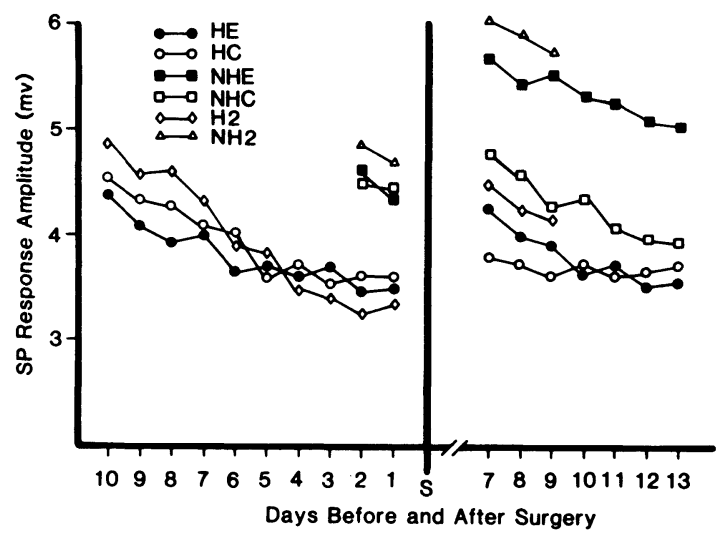

Figure 3. Mean amplitude of SP responses to the anditory stimulus before and after surgery for the habituation experimental sub. group (HE), the habituation control subgroup (HC), the nonhabituation experimental subgroup (NHE), and the nonhabituation control subgroup (NHC) in Experiment 1. $N$ was 9 for all of these subgroups, except for the HE subgroups which had an $N$ of 8. Mean amplitude of SP responses for the habituation (H2) and nonhabituation (NH2) groups in Experiment 2 are also given. Ns were 7 and 6, respectively. 


\section{EXPERIMENT 2}

Because the experimenter held all rats before and after surgery in Experiment 1, it is possible that he may have inadvertently influenced the amplitude of SP responses. This could have been particularly important in the effects of preoperative habituation of SP responses on the amplitude of SP responses after surgery. A control experiment appeared to be warranted. Experiment 2 was a partial replication of Experiment 1 , with the experimenter holding the rats before surgery and a second person, also a male, holding them after surgery. The second person was unaware of the group to which each rat belonged.

\section{Method}

Subjects. Of 19 male Holtzman rats initially tested, 13 met the two criteria described in Experiment 1. Data from these 13 rats are reported. They ranged in weight from 345 to $380 \mathrm{~g}$ at the time of surgery.

Procedures. Surgery, SP recording, and all procedures were the same as in Experiment 1 except as noted. The rats were divided into two experimental groups, a preoperative habituation group and a preoperative nonhabituation group. They were matched as closely as possible on the basis of the amplitude of nonspecific response. Preoperative SP recordings from these two groups were then made in the same way as had the corresponding groups in Experiment 1.

The second person participating in this experiment was first given experience in handling other rats and holding them for SP recording. SP responses were also recorded from one of his fingers during this preliminary period until they habituated to the auditory stimulus and nonspecific responses were reduced to a minimum.

Beginning 4 days after surgery, the rats were handled daily by the second person. On Day 6, this person held each rat while SP nonspecific responses were recorded without presentation of the auditory stimulus. Testing with the auditory stimulus was begun on Day 7.

\section{Results}

Lesions. For the habituation group, the mean lesion size was $33.5 \%$. For the nonhabituation group, the mean was $34.8 \%$.

SP responses. The mean amplitude of SP responses before and after surgery for the habituation and nonhabituation groups is also given in Figure 3. Before surgery, the habituation group displayed significant habituation $[F(9,54)=4.89, p<.01]$.

As can be seen in Figure 3, preoperative habituation did not reduce the effect of prefrontal lesions on SP response amplitude. Before surgery, SP response amplitudes of the nonhabituation subgroup across Days 2 and 1 were not significantly different from those of the habituation group across Days 10 and 9 $[F(1,11)=0.93, p>.05$, unweighted means procedure]. But across Days 7-9 after surgery, SP response amplitudes of the nonhabituation group were significantly larger than those of the habituation group $[F(1,11)=12.32, p<.01]$.

Because this effect of preoperative habituation was the same as that obtained in Experiment 1, it seems clear that the results of Experiment 1 were not seri- ously affected by the experimenter's holding the rats during SP recording both before and after surgery.

\section{EXPERIMENT 3}

In Experiments 1 and 2, it was assumed that the reduction in amplitude of the SP response to the auditory stimulus across days was due to habituation. SP response reduction could, however, have been due to some other factor, such as a fatigue process or damage to skin tissue from repeated application of the electrodes. This seemed unlikely, but it appeared that another control experiment would be useful.

If reduction in SP response amplitude across days was due to habituation, and not to some extraneous factor, then dishabituation should occur to a novel stimulus. This was tested in Experiment 3. The novel stimulus was a mild tailshock.

\section{Method}

Subjects. Data from eight male Holtzman rats are reported. They ranged in weight from 330 to $365 \mathrm{~g}$. They were selected from a group of 12 rats on the basis of the criteria described in Experiment 1.

Procedures. The procedures were the same as those used before surgery in Experiments 1 and 2, with the following exceptions. The rats were handled and then held during recordings by the same person who held the rats after surgery in Experiment 2. The equipment was operated by the experimenter or an experienced third person. The tailshock, $0.5 \mathrm{~mA}$ presented for $0.5 \mathrm{sec}$, was produced by a Lafayette Master Shocker controlled by a Hunter timer. The shock electrodes were silver disks, $6 \mathrm{~mm}$ in diameter, with a thin coating of saline-bentonite paste. They were mounted on the tail about $10 \mathrm{~mm}$ apart and at least $30 \mathrm{~mm}$ distal to the SP tail electrode. These electrodes were positioned on the tail each time the SP electrodes were applied.

SP responses to the auditory stimulus were first recorded at seven daily sessions. On Day 8 , the tail shock was presented one time, about 3 to $4 \mathrm{~min}$ after the recording had begun. About 5 to 7 min later, and after SP nonspecific activity had decreased to near the preshock level, the auditory stimulus was presented. The rats were then tested on a 9th day with only the auditory stimulus presented.

\section{Results}

The mean amplitude of SP responses to the auditory stimulus on the 9 test days is shown in Figure 4. There was a significant decrease in SP response amplitudes across Days 1-7 $[\mathrm{F}(6,42)=3.27, \mathrm{p}<.05]$. There was also a significant increase in response amplitudes across Days 6-9 $[F(3,21)=6.35, p<.01]$. These results suggest that the tailshock dishabituated SP responses on Days 8 and 9 and that, therefore, the decrease in response amplitudes across Days 1-7 was due to habituation. A similar reduction in SP responses before surgery in Experiments 1 and 2 was probably also due to habituation.

\section{DISCUSSION}

In Experiment 1, prefrontal lesions increased the amplitude of SP responses to an auditory stimulus in 


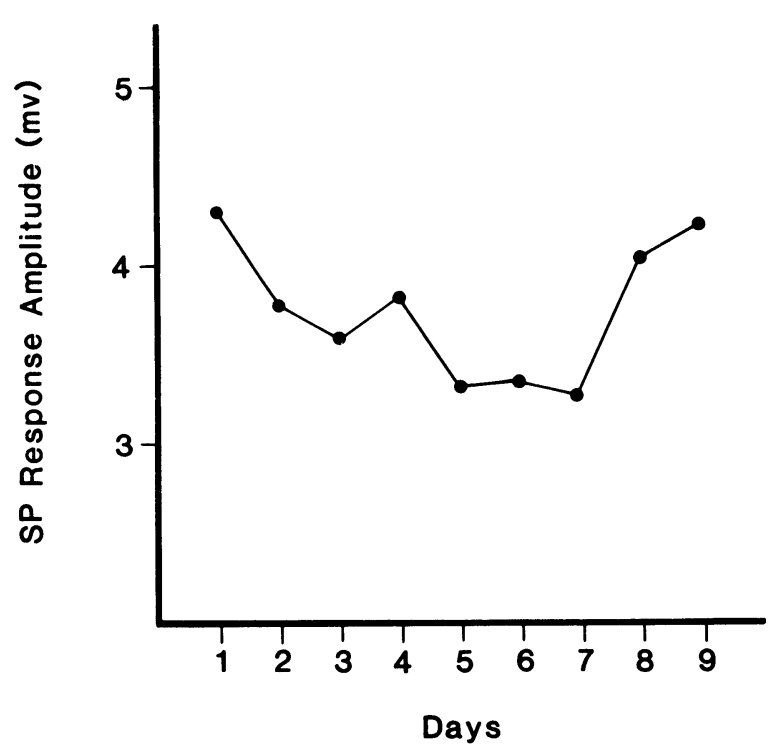

Figure 4. Mean amplitude of SP responses to the auditory stimulus across days. On Day 8, tallshock was given before the auditory stimulus was presented. $\mathbf{N}$ was 8.

the rat. If SP responses were habituated across days before surgery, prefrontal lesions disrupted the habituation. But the postoperative increase in the amplitude of SP responses was considerably less than that observed with rats who were not habituated before surgery. This indicated that the preoperative habituation of SP responses reduced the effects of prefrontal lesions. These results agree with those by Glaser and Griffin (1962) with heart rate in the rat.

In Experiment 2, this effect of preoperative habituation was also obtained when the experimenter held the rats during SP recording before surgery and a second person held them after surgery. This indicated that the results of Experiment 1 were not distorted by the experimenter's holding the rats both before and after surgery. Furthermore, the results of Experiment 3 demonstrated that the reduction in the amplitude of SP responses during daily presentations of the auditory stimulus could be disrupted by presentation of a novel stimulus, mild tailshock. This was probably due to dishabituation, and it suggests that reduction in SP responses to the auditory stimulus before the novel stimulus was presented was due to habituation. This also suggests that a similar reduction in SP responses to the auditory stimulus before surgery in Experiments 1 and 2 too was due to habituation.

It should be noted that habituation of SP responses across days before surgery in Experiments 1 and 2 could have reflected habituation to the recording situation as well as to the auditory stimulus. But it is likely that the habituation was mainly to the auditory stimulus, because habituation to the recording situation must have been occurring during the 3 adaptation days when the auditory stimulus was not presented. Nevertheless, this issue should be clarified by further investigation.

While it appears to have been established that the prefrontal cortex maintains an inhibitory influence on autonomic activity, the effect of preoperative habituation suggests two things about this inhibitory influence. First, the prefrontal cortex is part of an inhibitory system which includes other brain centers and pathways. Second, during habituation, inhibitory control of autonomic responses shifts at least partially from the prefrontal cortex to other parts of this system. The location of these other parts of the system is unclear, but it has been shown that electrical stimulation in several subcortical structures will inhibit heart rate and SP responses. These include the caudate nucleus and bulbar reticular formation (Wang \& Brown, 1956) and the septal area (Holdstock, 1967).

It is widely held that a reorganization or shift of function is responsible for behavioral recovery following lesions in some parts of the cortex (Meyer, 1973). But a shift of function from parts of the cortex to other brain structures in the normal animal seems to have received little attention. This problem deserves further study.

An important difference between results reported here and those obtained by Glaser and Griffin (1962) is that prefrontal lesions do not prevent habituation of an autonomic response. In Experiment 1, SP responses of the preoperative nonhabituation subgroup habituated after surgery. Roydes (1970) and Kolb (1974) have also found that skeletal responses of the rat will habituate after lesions in the frontal cortex, including the prefrontal region. It seems clear that the prefrontal cortex is not essential for habituation.

Even though the prefrontal cortex is not essential for habituation, its inhibitory influence could be important for habituation in the normal animal. However, the apparent shift in inhibitory control from the prefrontal cortex to other parts of the brain during habituation suggests that it may have a limited role in the habituation process.

\section{REFERENCES}

Brutkowski, S. Functions of the prefrontal cortex in animals. Physiological Review, 1965, 9, 721-746.

Glase R, E. M., \& Griffin, J. P. Influences of the cerebral cortex on habituation. Journal of Physiology (London), 1962, $160,429-455$.

HoLDsTock, T. L. Effect of septal stimulation in rats on heart rate and galvanic skin response. Psychonomic Science, 1967, 9, 37-38.

Kretpex, J. E., \& Price, J. L. The cortical projections of the mediodorsal nucleus and adjacent thalamic nuclei in the rat. Journal of Comparative Neurology, 1977, 171, 157-192.

KoLB, B. Some tests of response habituation in rats with discrete lesions to the orbital or medial frontal cortex. Canadian Journal of Psychology, 1974, 28, 260-267. 
MeYer, P. M. Recovery from neocortical damage. In G. M. French (Ed.), Cortical functions in behavior: Research and commentary. New York: Scott-Foresman, 1973.

Murry, M., \& Wang, G. A. Sweating in chronic rostocortical and caudocortical cats. Archives of Italian Biology, 1967, 39, 393-398.

Roberts, L. E., \& Youna, R. Electrodermal responses are independent of movements during aversive conditioning in rats, but heart rate is not. Journal of Comparative and Physiological Psychology, 1971, 77, 412-459.

RoYDEs, R. L. Frontal lesions impair habituations of the head shake response in rats. Physiology \& Behavior, 1970, 5, 1133-1139.

Wang, G. A., \& Brown, V. V. Suprasegmental inhibition of an autonomic reflex. Journal of Neurophysiology, 1956, 19, 564-572.

WilcotT, R. C. Effects of local blood removal on the skin resistance and potential. Journal of Comparative and Physiology Psychology, 1958, 51, 295-300.

WiLcotT, R. C. Cortical control of skin potential, skin resistance and sweating. Psychophysiology, 1967, 4, 500.

Wilcotr, R. C. Skeletal and autonomic inhibition from lowfrequency electrical stimulation of the cat's brain. Neuropsychologia, 1974, 12, 487-496.

(Manuscript received April 20, 1983;

revision accepted for publication July $19,1983$. 